Cinémas

Revue d'études cinématographiques

Journal of Film Studies

\title{
ALTMAN, Rick (direction). Sound Theory. Sound Practice. New \\ York : Routledge, 1992, 290 p.
}

\section{Jean Châteauvert}

Volume 4, numéro 3, printemps 1994

URI : https://id.erudit.org/iderudit/1001043ar

DOI : https://doi.org/10.7202/1001043ar

Aller au sommaire du numéro

Éditeur(s)

Cinémas

ISSN

1181-6945 (imprimé)

1705-6500 (numérique)

Découvrir la revue

Citer ce compte rendu

Châteauvert, J. (1994). Compte rendu de [ALTMAN, Rick (direction). Sound

Theory. Sound Practice. New York : Routledge, 1992, 290 p.] Cinémas, 4(3),

157-166. https://doi.org/10.7202/1001043ar d'utilisation que vous pouvez consulter en ligne.

https://apropos.erudit.org/fr/usagers/politique-dutilisation/ 
Altman, Rick (direction). Sound Theory. Sound Practice. New York: Routledge, 1992, 290 p.

Au cours des dernières années, différents ouvrages ont proposé de véritables panoplies d'outils d'analyse du son au cinéma. Pour n'en citer que deux, Yale French Studies y consacrait son numéro 60 en 1980 et plus récemment, en 1985, Elizabeth Weis et John Belton réunissaient une panoplie de travaux théoriques dans Film Sound. Theory and Pratice (New York: Columbia University Press). Ces ouvrages faisaient état de l'usage du son dans le cinéma narratif et proposaient différentes analyses de films. Sound Theory. Sound Pratice que dirige Rick Altman ${ }^{1}$ se présente d'entrée de jeu comme une alternative à ces premières publications en ce qu'il marque une ouverture sur d'autres genres de cinéma, notamment sur le cinéma dit "expérimental " et sur le cinéma des premiers temps, mais aussi en ce qu'il propose de nouvelles approches sur la place du son au cinéma, sur les concepts utilisés pour le décrire, sur les différents usages du son, sur le rapport au genre, etc.

Réunissant une panoplie de chercheurs, l'ouvrage se divise en trois grands volets qu'introduit Altman: en premier lieu, l'ouvrage propose quatre contributions qui interrogent les théories du son et avancent des alternatives théoriques pour repenser le son au cinéma; dans le second volet, consacré à l'histoire du son au cinéma, on peut lire trois articles qui repensent le développement du son au cinéma; dans le dernier volet, on retrouve cinq contributions consacrées à des "domaines négligés" par la recherche dont notamment la place de la femme au sein des voix narratives, le son dans les dessins animés, dans les documentaires, etc.

On le voit d'emblée, la somme que constitue Sound Theory. Sound Pratice est particulièrement vaste et ouvre de nouvelles 
perspectives d'analyse tant par les champs d'application convoqués que par les idées suscitées. Je me contenterai de souligner ici quelques-unes de ces nouvelles perspectives en insistant sur l'abondance et la diversité des enseignements que le lecteur y trouvera.

Dans son introduction générale, Altman propose de repenser tout le cinéma non plus comme un texte, mais comme un événement qui s'ancre dans une culture et au sein de laquelle il est inévitablement conduit à "dialoguer" avec d'autres pratiques culturelles, tels la radio, le disque, le théâtre, etc. (p. 13). Il propose ainsi de relire l'histoire du son au cinéma non plus comme une évolution progressive vers les pratiques actuelles, comme on peut souvent le lire, mais à l'opposé, depuis les expériences et les recherches esthétiques et techniques qui entourèrent son développement.

Dans son article consacré aux matériaux sonores, Altman propose une vision de l'histoire du son au sein de laquelle l'enregistrement du son génère non pas une simple reproduction, mais une interprétation, une version spécifique de l'événement sonore qui implique des choix, mais aussi une véritable orientation dans la perception du son (p. 27). Il formule à cette occasion une des grandes thèses qui parcourt ce livre, l'idée que la trame sonore du film est une "illusion de reproduction" qui masque une "représentation", un travail de mise en scène du son (p. 29) et que ce serait précisément dans ce décalage de la reproduction sur la représentation que reposerait le pouvoir du son au cinéma.

Ouvrant le volet consacré aux "Perspectives théoriques", Altman bat en brèche quatre idées courantes sur le son au cinéma: 1) la conception pseudo-historique qui date le son de 1927 avec The Jazz Singer de Crosland, le son étant présent au cinéma depuis ses premiers jours via le bonimenteur, la musique du pianiste ou de l'orchestre, pour ne pas mentionner les bruiteurs qui parfois agrémentaient les projections; 2) la définition ontologique du cinéma par l'image mouvante, comportant des cartons noirs, des images arrêtées, des fondus, etc.; 3) la reproduction du son est, comme on l'a souligné plus haut, illusoire; on ne peut jamais que représenter les sons; 4) le nominalisme des sons ne permet guère de les désigner; en fait on désigne le plus souvent la 
source sonore plutôt que le son; 5) le cinquième point correspond à une conception qui n'est qu'à demi fausse ou, si l'on préfere, à demi vraie: le son au cinéma a une valeur indexicale au regard de la réalité dans la mesure où il est enregistré - il y a une analogie directe entre le matériau et la réalité qu'il reproduit —, mais il s'en écarte cependant en ce qu'il est retravaillé, remodelé en studio (p. 35-45).

Dans son article consacré à l' "Espace sonore", Altman réitère sa distinction entre la reproduction et la représentation sonore en marquant cette fois une articulation entre le travail sur le son au cinéma et d'autres formes, tels la radio, le théâtre, le phonographe et le discours public (p. 47). Il apparaît ainsi que si certains penseurs tel Maxfield, qui a travaillé à développer la technologie du son, ont prôné une adéquation stricte entre les emplacements des microphones et des caméras, c'est avant tout un paramètre d'intelligibilité des voix qui s'est imposé (p. 53) sous l'influence de la radio et du cinéma muet (avec ses cartons pour le dialogue). Cette première scission entre le son et l'image s'accompagna d'une autonomisation du son qui devint progressivement une trame sonore continue tandis que la bande-images maintenait son caractère fractionné par le montage (p. 57). Toutefois, ce parti pris de clarté n'empêcha pas la création d'une certaine perspective sonore (point of audition) et l'on créa ainsi des effets de distance dans le rendu des sons, notamment par l'ajout de réverbération et une modalisation du volume sonore qui créent l'impression de proximité ou d'éloignement d'une source. Or, cette perspective sonore s'établit non pas en regard des personnages visualisés - en fait nous ignorons ce qu'ils entendent - , mais en fonction des spectateurs qui projettent leur expérience auditive sur celle des personnages (p. 61). Il apparaît ainsi que ce qu'on désigne comme le "réalisme sonore" résulte en fait de modèles de réalité qui préexistaient au cinéma, tels les modèles théâtral, radiophonique, etc. qu'expérimentaient déjà le spectateur, mais aussi de codes qui se sont forgés sur cette nouvelle technologie qui combinait un son et une image (p. 64).

Dans son travail sur le rendu sonore, James Lastra reprend la distinction entre la reproduction du son et sa représentation. Il soumet ainsi que les pratiques de reproduction sonore ne sont 
pas " naturelles " mais construites, élaborées à partir de modèles narratifs (p. 70). Au sein du film, la valeur narrative du son prédomine et, partant, module d'entrée de jeu le traitement du son en privilégiant l'intelligibilité du son au détriment du réalisme spatial (p. 77). Lastra voit ainsi, dans le traitement qui privilégie l'intelligibilité de la voix, l'influence du théâtre et de la radio mais aussi, et l'idée mérite d'être soulignée, celle du cinéma muet, qui a progressivement déterminé le rôle et la fonction du discours verbal dans un récit filmique (p. 80). La fidélité apparaît dans ce contexte non plus comme une donnée, mais comme une résultante de la synchronisation d'un son et d'une source à l'image. Le théoricien en vient enfin à mettre en doute le concept même de "son original ", le son au cinéma étant davantage un construit de la représentation qu'une véritable reproduction d'un événement sonore.

Dans son article consacré à l'analyse du matériel sonore, Steve Wurtzler affine les distinctions courantes entre le son live, c'està-dire le son direct, et le son enregistré. Il propose ainsi un système à double entrée suivant que le son est reproduit de façon simultanée ou postérieur à son émission et suivant que sa source est, en terme spatial, présente ou absente. Il distingue ainsi quatre configurations: 1) le son direct dont la source est présente tant en termes spatial que temporel; 2) le son dont la source est absente sur le plan spatial mais dont l'émission est contemporaine de son écoute, tel le son au téléphone, à la télévision ou à la radio ; 3) la source où plus exactement la source apparente peut être présente dans le lieu physique encore que le son soit en fait en différé, à l'instar du chanteur qui mime une chanson préenregistrée (playback); 4) enfin, il y a le son enregistré qui se présente pour tel, soit un son dont la source est absente à la fois du lieu et du moment où il est reproduit (p. 89). Invoquant des exemples télévisuels, tels les journalistes en studio qui commentent des événements en direct, et le caractère aléatoire du sens de l'adjectif live lorsque l'on qualifie un enregistrement musical (p. 93), Wurtzler interroge l'évolution du cinéma depuis la volonté de reproduire l'événement sonore vers la création d'un pseudo-événement où l'on cherche avant tout à préserver l'intelligibilité des voix. L'article se termine par une 
réflexion extrêmement stimulante sur l'existence même d'un son "original" et propose une approche du son au cinéma non plus comme une donnée préalable, mais comme un produit du film (p. 101).

Cette première partie théorique se clôt avec un court article de Michel Chion qui reprend quelques thèses de son livre $\mathrm{La}$ Toile trouée.

La seconde partie du livre est consacrée à l'histoire du son au cinéma. Altman introduit les différents articles de ce volet historique en établissant toute une série de parallèles entre le cinéma et d'autres formes, tels la photographie, le théâtre, la radio, etc., pour repenser les différentes fonctions du son dans les films (p. 114 et suivantes). L'exercice s'avère particulièrement stimulant et marque une ouverture sur une nouvelle conception de l'histoire du cinéma au sein de laquelle l'identité et la fonction sociale du médium du cinéma peut varier - comme lorsque le cinéma "muet" est devenu sonore —, une histoire où le développement de ce que l'on proposa d'appeler à l'époque l'audien (c'est-à-dire "ce qui permet d'être sonore") (p. 125) n'est pas une droite continue, mais une série de mouvements complexes où la technique cinématographique gagnait sur un point ce qu'elle perdait sur un autre - l'avènement du son a ainsi paralysé les mouvements et les emplacements des caméras pendant quelque temps en raison de la proximité que nécessitaient les microphones - , une histoire enfin où il n'y a pas une évolution continue dans la définition du réalisme, mais en lieu et place des luttes et des influences diverses qui ont leurs racines dans les différents modèles que furent la radio, le disque, le théâtre, le téléphone, etc. (p. 122).

Ouvrant les travaux de ce volet historique, Alan Williams attaque les visions téléologiques de l'histoire du cinéma et rappelle que dès l'exposition Universelle de 1900, on parvenait à synchroniser des images et du son et que dès 1910, grâce à un système de compresseur d'air, on pouvait amplifier le son pour fournir une salle pouvant contenir 5000 personnes $^{2}$ (p. 127). L'habitude de la représentation avec un commentateur, le "bonimenteur", était cependant plus forte et le cinéma conserva son statut "muet" pendant nombre d'années. En fait, l'avènement du son enregistré 
fut provoqué par les maisons de production parce que, et la thèse ne manque pas d'intérêt, le film sonore leur permettait de réduire le caractère labile des représentations, largement déterminées par la qualité du conférencier qui commentait le film (p. 129). Le choix des spectateurs tenait alors du film présenté, mais aussi de la prestation du bonimenteur, lequel pouvait tantôt transformer une simple production en la plus belle histoire qui soit, ou à l'inverse, ruiner un film par un commentaire déficient. Mais la « révolution du parlant" n'aurait sans doute pas été aussi rapide n'eût été le succès du fameux Jazz Singer. Ce succès, Williams l'explique par la nature duelle de ce film qui réunit un véritable spectacle musical sur scène et une histoire que ponctue, çà et là, une musique d'accompagnement; ce serait précisément cette combinaison de ces deux genres de spectacles qui aurait remporté la faveur populaire.

Reprenant les thèses de Bordwell sur le cinéma hollywoodien, Williams souligne que, déjà au sein du cinéma muet, il était des films qui accordaient une place prépondérante au dialogue au regard d'autres films qui privilégiaient une esthétique visuelle (p. 132). En ce sens, l'avènement du son eut l'effet d'un véritable catalyseur qui provoqua l'éviction de certains genres, tels les mélodrames, où prévalait l'expression corporelle des sentiments au profit d'autres genres, où primait le dialogue (p. 136). L'avènement du son au cinéma s'accompagna par ailleurs d'un ancrage réaliste et culturel lié à la langue, qui rompait avec le réalisme esthétique du cinéma muet : le son se traduisit ainsi par des frontières culturelles plus marquées entre les films des divers pays, frontières que la postsynchronisation permit de rendre plus perméables en réintroduisant des voix à mi-chemin entre la réalité et l'abstraction (p. 136).

Suit un article de Natasia Durovicova sur les versions étrangères des productions américaines entre 1929 et 1933 (Foreign Langage Version) : au cours de ces années où les lois sur les importations et les exportations de films étaient particulièrement strictes, on ne pouvait exporter un film dans un pays, tels la France ou l'Italie, qu'à la condition de retourner le film en studio dans le pays visé avec des acteurs locaux, les sous-titres étant alors rejetés parce que rattachés au cinéma muet (p. 138). On 
n'accepta que progressivement les films "doublés", acceptation rendue possible, suivant Durovicova, parce que l'on construisit une nouvelle représentation du corps dans l'espace fictionnel et parce que le langage devint progressivement un instrument privilégié pour familiariser le spectateur avec le monde visualisé (p. 152).

Explorant le passage au son magnétique, John Belton souligne que si le son magnétique offre une meilleure qualité technique que le son optique, ce dernier fut néanmoins taxé d'irréalisme à ses débuts dans les années 1952-1954, habitué que l'on était à son prédécesseur (p. 156). Pareillement, le son stéréophonique, associé aux productions importantes, généralement tournées dans des formats marqués, différents, tel le cinémascope, passa d'abord pour un son spectaculaire, entendre irréaliste, et à ce titre reçut un accueil mitigé (p. 159). Il faut dire que la technologie du son stéréophonique, alors limitée à quatre haut-parleurs, accusait des passages brusques d'un haut-parleur à un autre, saute qu'atténua l'ajout de nouveaux haut-parleurs (p. 162). En fait, le maintien d'un rendu sonore monodique se perpétua tardivement au-delà des années soixante et tint lieu de point de repère aux spectateurs qui expérimentaient de nouveaux formats d'écran et de nouvelles pellicules.

Après quoi s'ouvre la dernière grande section de ce recueil, et j'ajoute, sans doute la plus inégale de toutes, consacrée aux "Domaines négligés" par la théorie du cinéma. Introduite encore une fois par Altman qui souligne le peu d'études consacrées au cinéma du tiers monde, aux petites productions, aux films de court métrage, au film d'animation, etc., cette dernière partie se veut être une occasion pour soulever de nouvelles questions, de nouvelles problématiques.

Amy Lawrence interroge ainsi la place de la femme dans la production cinématographique des pays du tiers monde et propose une étude approfondie du film Surname Viet. Given Name (Trinh Minh-ha, 1989) qui entremêle la fiction et le documentaire. À défaut de convaincre entièrement le lecteur, on se doit de souligner la perspicacité de cette analyse qui ne manque pas de susciter des idées sur le concept de fiction, sur la vérité et le mensonge et sur la place de la femme au cinéma. 
Scott Curtis propose, pour sa part, de repenser les catégories d'analyse diégétique/non diégétique, la hiérarchie entre image et son et même le distinguo "parole, bruit, musique" en analysant le travail sonore dans les premiers dessins animés (cartoons) de la Warner (p. 192). Curtis rappelle ainsi que l'avènement du son au cinéma s'est traduit par des politiques de fusion entre les industries du disque et du cinéma; l'on vit naître du même coup de nouvelles structures de production tel Merrie Melodies ou Looney Tunes, qui avaient pour tâche de réaliser des dessins animés fondés sur des pièces musicales dont les droits étaient détenus par la compagnie mère (p. 194). Après avoir retracé les différentes stratégies de création, suivant que l'on se fondait sur un rythme particulier, sur un thème ou sur un sujet, Curtis remarque que le film d'animation était à cette époque conçu comme un produit de divertissement (entertainment) qui se greffait au véritable produit culturel qu'est le film.

Interrogeant la valeur d'une occurrence sonore, Curtis souligne encore qu'une même occurrence peut assumer différentes fonctions et que, suivant le contexte et l'interprétation du spectateur, un même son peut être perçu comme un bruit, comme de la musique, voire comme une parole (p. 196). De là, Curtis propose de distinguer l'usage isomorphique du son lorsqu'il y a des correspondances étroites entre le son et l'image, tant et si bien qu'ils sont indissociables, de l'usage iconique lorsque l'analogie entre le son et l'image n'est que partielle, limitée à certaines dimensions (p. 201). Et l'auteur de remarquer que ce ne sont pas des catégories exclusives, mais plutôt des registres de relations qui peuvent fonctionner de concert ou de façon isolée (p. 202). Enfin, interrogeant le statut indexical du son au sein du cinéma courant, c'est-à-dire la corrélation obligée entre un son et une source, Curtis en arrive à relativiser ces corrélations en soulignant que tant dans les dessins animés que dans le cinéma courant, la valeur d'une même occurrence sonore peut être des plus variables suivant son contexte d'occurrence et la relation qui s'établit entre le son et l'image.

Mary Pat Klimek développe une approche comparative entre les usages du son au théâtre et au cinéma en confrontant différentes adaptations cinématographiques de pièces de Shakespeare (p. 209). Si l'on peut regretter le manque de distance théorique 
dans cet article, on se doit d'en souligner la qualité de l'analyse comparative entre les différentes adaptations, analyse qui se révèle l'occasion de proposer des alternatives possibles dans l'utilisation du son au cinéma.

Jeffrey K. Ruoff interroge ensuite les conventions du son dans le genre du documentaire et plus particulièrement dans la télésérie documentaire An American Family (Gilbert) des années soixante-dix. Il développe ainsi une approche comparative entre les différents usages du son dans le documentaire au regard du film de fiction et analyse les paramètres qui conditionnent le choix des intervenants, soit le casting des voix dans les deux genres (p. 222). De là, Ruoff propose une analyse du rôle de la musique et du commentaire en voix over dans les documentaires et dans les films de fiction pour en conclure que tout film, aussi objectif qu'il soit, est d'abord et avant tout un construit qui repose sur des stratégies discursives et des conventions (p. 234).

Andrea Turpin propose la dernière analyse, qui est consacrée aux films d'Andrei Tarkovsky: Le Sacrifice, Nostalgia, Stalker et Le Miroir. Elle y souligne l'utilisation spécifique du son chez ce cinéaste qui module le traitement sonore pour en quelque sorte transcender la réalité matérielle du monde filmé et lui ajouter une dimension irréelle, spirituelle (p. 239).

Enfin, dans un retour sur l'abondance des recherches proposées, Altman fait un bref rappel des concepts qui ont été proposés ou affinés en cours de route et propose un aide-mémoire précieux qui renvoie aux différents textes avant de proposer une bibliographie substantielle sur le son au cinéma.

Sur un plan théorique, on le voit à travers ce trop court résumé, les thèses que l'on propose dans ce recueil sont très stimulantes, tant par leur diversité que par les remises en question qu'elles suscitent. Il est ainsi des idées sur les genres, sur les catégories d'analyse, sur les films qui, à défaut de provoquer une adhésion directe du lecteur - je pense notamment à la remise en question de concepts tels que les sons diégétique/ extradiégétique qui confond, me semble-t-il, l'application des concepts et les distinctions qu'ils permettent - , ne manqueront pas de susciter nombre de réflexions chez tous ceux qui s'intéressent à la question du son au cinéma. 
On peut cependant reprocher à ce recueil de réunir des articles qui font état de recherches générales sur la question du son et des articles qui ont une valeur extrêmement ponctuelle, circonscrite à une analyse de film ou à une discussion théorique. Ainsi, si les articles d'Altman, Lastra, Williams ou Curtis s'imposeront comme des références sur le travail du son au cinéma, il en est d'autres qui présentent un intérêt beaucoup plus limité, circonscrit en quelque sorte à un genre précis, voire à un seul film. Mais malgré ce caractère variable, ou plutôt à cause de lui, la qualité des analyses et des réflexions théoriques que l'on y trouve est telle que ce livre ne manquera pas d'intéresser tous ceux et celles qui s'intéressent à la question du son au cinéma, mais aussi l'étudiant ou le professeur qui est à la recherche d'une analyse de la trame sonore chez tel cinéaste ou dans tel film. Aussi, si Sound Theory. Sound Pratice n'est pas l'ouvrage qui clôt la réflexion sur le son, il ouvre à une réflexion à laquelle tous ceux et celles qui s'intéressent à la question du son au cinéma devraient prêter une oreille attentive. À défaut d'en sortir convaincu, on ne manquera pas d'y trouver matière à réflexion.

\section{NOTES}

1 Les gens qui s'intéressent à la question du son au cinéma connaissent bien Rick Altman pour ses articles publiés notamment dans le numéro spécial de Yale French Studies et dans la revue Iris, $\mathrm{n}^{\circ} 3$ (1985) et $\mathrm{n}^{\circ} 4$ (1986). On trouve une bibliographie développée à la fin du volume.

2 En revanche, on peut s'interroger sur l'intelligibilité du dialogue dans un tel système, cette question ayant été au cœur des recherches sur le son jusque dans les années vingt.

JeAn Châteauvert

Université de Montréal 